\title{
Rendimiento de Enfoques de Control Auto-Disparado
}

\section{(Performance of Self-Triggered Control Approaches)}

\author{
Carlos Rosero ${ }^{1}$, Cristina Vaca ${ }^{1}$, Luz Tobar Subía ${ }^{1}$, Fredy Rosero ${ }^{1}$
}

\begin{abstract}
Resumen:
El control auto-disparado produce secuencias de muestreo no periódico que varían según varios de factores de diseño relacionados con la estabilidad y el rendimiento del sistema a ser controlado. Dentro de este marco, recientemente han sido desarrollados dos enfoques dirigidos a minimizar un costo cuadrático, considerando un rendimiento óptimo y persiguiendo el mismo objetivo de control; cada una de ellos sigue una regla de muestreo diferente. Un enfoque se basa en mantener el valor de control actual tanto tiempo como sea posible, mientras que un umbral de rendimiento óptimo no se traspase. El otro enfoque se basa en la generación de una señal de control a trozos que se aproxima a una señal de control óptima continua, sujeta a determinadas limitaciones. Este artículo presenta un estudio comparativo entre los dos enfoques, proporcionando una percepción útil para la realización de futuras investigaciones. Como métricas de interés, el rendimiento de control y la utilización de recursos fueron considerados, y para evaluarlos, se hizo uso del intervalo de muestreo promedio y del costo normalizado. Se demostró que el diferente espacio de búsqueda de cada enfoque plantea un desafío para diseñar un marco de comparación equitativo, y que ambos enfoques superan al muestreo periódico.
\end{abstract}

Palabras clave: procesamiento en tiempo real; reglas de muestreo; control óptimo; control manejado por eventos; regulador cuadrático lineal.

\begin{abstract}
:
The self-triggered control produces non-periodic sampling sequences that vary depending on design factors related to stability and performance of the controlled system. Within this framework, two approaches aimed at minimizing a quadratic cost have been developed recently, considering an optimal performance and pursuing the same control objective; each approach follows a different sampling rule. One approach is based on maintaining the current control value as long as possible, while an optimal performance threshold is not passed. The other approach is based on the generation of a piecewise control signal, which approximates a continuous optimal control signal subject to certain constraints. This article presents a comparative study between the two approaches, providing a useful insight for conducting future research. Control performance and resource utilization were considered as metrics of interest and to evaluate them, the average sampling interval and the standardized cost were taken into account. It was shown that the different search space of each approach poses a challenge to design an equitable framework of comparison, and that both approaches exceed the periodic sampling.
\end{abstract}

Keywords: real-time computing; sampling rules; optimal control; event-driven control; linear quadratic regulator.

\footnotetext{
${ }^{1}$ Universidad Técnica del Norte, Ibarra - Ecuador ( \{cxrosero, cvaca, Imtobarsubia, farosero\} @utn.edu.ec )
} 


\section{Introducción}

En la actualidad, los controladores son implementados usando sistemas computacionales en los cuales la tarea de control comparte recursos de procesamiento y red con otras tareas. El muestreo periódico establece que la tarea de control se debe ejecutar (disparar) en función del tiempo, es decir, cada vez que el período de muestreo se cumple, incurriendo a menudo en la sobreutilización de recursos (Astrom, 1999). Sin embargo, los esquemas no periódicos de muestreo pueden ser más efectivos que los periódicos, al balancear el rendimiento de control en lazo cerrado (calidad de control) en función de la utilización de recursos (Gommans, 2015).

Dos tipos de aproximaciones que abandonan el paradigma de control periódico son el control disparado por eventos (ETC, Event-Triggered Control) y el control auto-disparado (STC, SelfTriggered Control), (Heemels, 2012). El primero es reactivo y requiere de la ejecución de la tarea de control cuando algún estado de la planta sobrepasa cierto valor de umbral (Arzén, 1999). El segundo calcula previamente cuándo será la próxima vez que la tarea de control se debe ejecutar (Velasco, 2003). Tanto ETC como STC se componen de dos elementos: un controlador con realimentación que calcula la entrada de control, y un mecanismo de disparo que determina cuándo debe actualizarse de nuevo esta entrada de control.

El presente artículo se centra en analizar reglas para muestreo aplicadas en STC, direccionadas a producir secuencias de entradas de control con costo menor o igual que el costo óptimo, el cual corresponde al mínimo dado por el regulador cuadrático lineal (LQR, Linear Quadratic Regulator) de tiempo discreto (Astrom, 1997). El problema de la regla para muestreo óptimo ha sido recientemente abordado en (Rabi, 2008), (Velasco, 2011), (Meng, 2012), (Molin, 2013), (Bini, 2014), (Gommans, 2014) y (Velasco, 2015), cuya contribución ofrece formulaciones factibles en diferentes entornos, a la vez que identifica posibles limitaciones en términos de optimalidad, tratabilidad computacional y/o aplicación práctica. Sin embargo, dentro de la literatura existente, no se encuentra un marco comparativo o una metodología para evaluar sus propiedades.

Para superar esta limitación el presente trabajo considera dos enfoques de STC, (Gommans, 2014) y (Velasco, 2015), con el objetivo de identificar los aspectos claves que una metodología de comparación debe tener en cuenta para la evaluación del rendimiento. La contribución de este trabajo consiste en presentar un análisis comparativo de las dos aproximaciones, donde el rendimiento de control y la utilización de recursos son las principales métricas de interés. Además, el equilibrio inherente entre rendimiento y utilización es la base para establecer la metodología utilizada en el análisis comparativo de desempeño. Los resultados obtenidos a través de simulaciones revelan sus posibles beneficios y limitaciones, a la vez que permiten extraer lineamientos para el diseño futuro de nuevas reglas de muestreo óptimo. 
El resto del documento está estructurado de la siguiente manera. La Sección 2 presenta la base teórica necesaria para comprender los dos enfoques de control; muestra las pautas a seguir para desarrollar el análisis comparativo. La Sección 3 ilustra numéricamente la evaluación de rendimiento y muestra la discusión de los resultados. Finalmente, la Sección 4 concluye el manuscrito.

\section{Metodología}

En adelante, la aproximación en Gommans (2014) llamada control cuadrático lineal autodisparado (Self-Triggered Linear Quadratic Control) se citará como STLQ. La aproximación en Velasco (2015), llamada control auto-disparado inspirado en muestreo óptimo (Optimal-SamplingInspired Self-Triggered Control), se mencionará como OSIST.

Ambos enfoques de STC consideran un sistema lineal continuo invariante en el tiempo (LTI, Linear Time-Invariant), regido por

$$
\left\{\begin{array}{c}
\dot{x}=G x+H u \\
x(0)=x_{0}
\end{array}\right.
$$

donde $x \in \mathbb{R}^{n}$ es el vector de estados y $\bar{u} \in \mathbb{R}^{m}$ es el vector de señales de entrada, $G \in \mathbb{R}^{n \times n}$ y $H \in \mathbb{R}^{n \times m}$ son matrices que describen la dinámica del sistema, y $x_{0}$ es el vector de estados iniciales.

Una diferencia fundamental entre STLQ y OSIST, consiste en que el primero contempla el trabajo bajo condiciones de ruido. Sin embargo, el estudio desarrollado a través de este artículo no lo considera, ya que ambos enfoques presentan contribuciones comparables para el caso de condiciones ideales.

La señal de entrada de control continua $u$ en (1) está limitada a ser constante a trozos,

$$
u(t)=u_{k}, \forall t \in\left[t_{k-1}, t_{k}\right),
$$

donde $u_{k} \in \mathbb{R}^{m}$ es la entrada de control en tiempos discretos $k \in \mathbb{N}$, y $t_{k} \in \mathbb{R}$ simboliza los instantes de muestreo. El patrón de muestreo está representado por los valores que separan dos instantes consecutivos llamados intervalos de muestreo $\tau_{k}$. Los instantes de muestreo y los intervalos de muestreo están relacionados entre sí a través de

$$
\left\{\begin{array}{c}
t_{k}=t_{0}+\sum_{i=0}^{k-1} \tau_{i}, t_{0}=0, \quad k \geq 1 \\
\tau_{k}=t_{k+1}-t_{k}
\end{array}\right.
$$

El problema óptimo LQR de horizonte infinito en tiempo continuo contempla la búsqueda de una señal de entrada que minimiza la función de costo

$$
J=\int_{0}^{\infty}\left(x^{T} Q x+u^{T} R u\right) d t
$$


considerando las matrices $Q \in \mathbb{R}^{n \times n}$ positiva semidefinida, y $R \in \mathbb{R}^{m \times m}$ positiva definida. Para el control LQR de horizonte infinito en tiempo discreto, se pueden usar formas discretas conocidas (Astrom, 1997).

En el muestreo periódico se tiene $\tau_{k}=\tau$ para todos los $k$, donde $\tau$ es el período de muestreo. A través de técnicas de discretización usuales (Astrom, 1997), y considerando

$$
\begin{array}{r}
\Phi(\tau)=e^{G \tau}, \quad(5) \\
\Gamma(\tau)=\int_{0}^{\tau} e^{G(\tau-t)} d t H,
\end{array}
$$

la dinámica (1) puede ser descrita por el sistema LTI en tiempo discreto

$$
\left\{\begin{array}{c}
x_{k+1}=\Phi(\tau) x_{k}+\Gamma(\tau) u_{k} \\
x(0)=x_{0}
\end{array}\right.
$$

donde $x_{k}=x\left(t_{k}\right)$ es el estado muestreado en $t_{k}$. La ecuación (4) puede describirse como un problema óptimo LQR de horizonte infinito en tiempo discreto,

$$
J_{\tau}=\sum_{0}^{\infty}\left(x_{k}^{T} Q_{\tau} x_{k}+2 x_{k}^{T} S_{\tau} u_{k}+u_{k}^{T} R_{\tau} u_{k}\right)
$$

con las matrices $Q_{\tau} \in \mathbb{R}^{n \times n}$ positiva semidefinida, $R_{\tau} \in \mathbb{R}^{m \times m}$ positiva definida y $S_{\tau} \in \mathbb{R}^{n \times m}$, todas obtenidas a través de discretizaciones conocidas (Astrom, 1997).

\subsection{Enfoques de control auto-disparado}

En STLQ y OSIST, tanto la regla de muestreo como la entrada de control a trozos se resuelven minimizando el costo LQR. La regla de muestreo genera intervalos de muestreo $\tau_{k}$ según el estado muestreado $x_{k}$ y un conjunto de parámetros. La entrada de control consiste en una ganancia calculada para trabajar durante el intervalo $\tau_{k}$ y depende además del estado muestreado $x_{k}$.

\subsubsection{Control Cuadrático Lineal Auto-Disparado (STLQ)}

Esta aproximación presenta una regla de muestreo inspirada en el problema clásico LQR de tiempo discreto que busca mantener constante cada entrada de control durante el mayor número de pasos posible, ampliando así cada intervalo de muestreo, y garantizando un rendimiento de control (Gommans, 2014). Esta garantía de rendimiento se da en términos de una función de costo LQR de tiempo discreto; las muestras se activan de acuerdo con una degradación permisible, especificada en el costo LQR y limitada por un intervalo de muestreo mínimo dado.

La regla de muestreo puede ser descrita a través de una notación diferente a la original, así 


$$
\tau_{k}=\max \left\{\tau_{c}:=c \tau_{\min }, c \in \mathbb{N}^{+} \mid \min \left[J_{\tau_{c}}\left(u, \tau_{\min }\right)\right] \leq \beta x_{k}^{T} P_{\tau_{\text {min }}} x_{k}\right\},
$$

considera una función de costo

$$
J_{\tau_{c}}\left(u, \tau_{\min }\right)=\int_{0}^{\tau_{c}}\left(x^{T} Q x+u R u\right) d t+x_{k+1}^{T} P_{\tau_{\min }} x_{k+1}
$$

El cálculo de $\tau_{k}$ en (9) busca maximizar el intervalo de muestreo actual $\tau_{c}$ (múltiplo del intervalo de muestreo mínimo $\tau_{\min }$ dado) durante el cual se aplica una señal de control constante $u$. El valor específico de $u$ debería incurrir en un costo (10) menor que $\beta$ veces el costo mínimo que obtendría un controlador periódico LQR de tiempo discreto, con intervalo de muestreo $\tau_{\text {min }}$, donde $\beta \geq 1$. En (9), $P_{\tau_{\min }}$ es la única solución semidefinida positiva a la ecuación algebraica discreta de Riccati (DARE, Discrete Algebraic Ricatti Equation) del costo cuadrático discreto (8) para $\tau_{\min }$ periódico. El primer sumando en (10), el término integral, es el costo cuadrático en tiempo continuo (4) sobre el intervalo de muestreo actual. El segundo sumando en (10), es el costo al suponer que las muestras restantes, después del intervalo de muestreo actual, se realizan periódicamente con $\tau_{\min }$.

Además, esta aproximación sugiere que la señal de control, para un intervalo de muestreo dado $\tau_{k}$, es descrita por

$$
u_{k}=\underset{u}{\arg \min }\left[J_{\tau_{c}}\left(u, \tau_{\min }\right)\right]
$$

En el documento original (Gommans, 2014) se proporciona una expresión alternativa para (11) que tiene una forma estándar de retroalimentación lineal de estados $u=Z\left(\tau_{k}, \tau_{\min }\right) x_{k}$ donde la ganancia $Z(\cdot)$ cambia en cada ejecución del controlador. Sin embargo, por propósitos de comparación, la expresión dada en (11) ha sido considerada la más adecuada.

\subsubsection{Control Auto-Disparado Inspirado en Muestreo Óptimo (OSIST)}

Establece una regla de muestreo que genera entradas de control de tal manera que la señal de control a trozos resultante, se aproxima a la entrada de control LQR de tiempo continuo, proporcionando un muestreo más denso cuando la señal de control exhibe más variación. La garantía de rendimiento se da en términos de densidad de muestreo, definida como el número de muestras a lo largo de un intervalo de tiempo (véase (Bini, 2014)). Las muestras se disparan de acuerdo con la derivativa de la entrada de control LQR de tiempo continuo y tienen como límite superior un valor máximo dado.

La regla de muestreo se sintetiza como 


$$
\tau_{k}=\tau_{\max } \frac{1}{\frac{\tau_{\max }}{\eta}\left|Z(G+H Z) x_{k}\right|^{\alpha}+1}
$$

donde $\tau_{\max }$ es el límite superior para los intervalos de muestreo, $\eta$ determina la densidad de la secuencia de muestreo (pequeños valores de $\eta$ producirán instantes de muestreo más densos, es decir muestras más frecuentes, y viceversa). El término $Z$ es la ganancia óptima de retroalimentación en tiempo continuo, obtenida al minimizar el costo cuadrático continuo (4). El parámetro $\alpha \geq 0$ determina la distribución del conjunto de muestras, permitiendo la aparición de más muestras cuando la derivativa de la entrada de control LQR en tiempo continuo es mayor. Véase (Bini, 2014) para una discusión sobre los ajustes del parámetro $\alpha$.

Además, este enfoque sugiere que la señal de control a trozos a ser aplicada en (2) para un intervalo de muestreo dado $\tau_{k}$, es descrita por

$$
u_{k}=\arg \min \left[J_{\tau_{k}}\left(u, \tau_{k}\right)\right]
$$

que es la señal de control óptima a trozos que se aplicaría al suponer un muestreo periódico $\operatorname{con} \tau_{k}$. La señal de control en (13) también puede expresarse usando una forma estándar de retroalimentación lineal $u=Z\left(\tau_{k}\right) x_{k}$ donde la ganancia $Z(\cdot)$ cambia en cada ejecución del controlador y corresponde a la ganancia de retroalimentación óptima en tiempo discreto, encontrada al minimizar el costo (8) para un período de muestreo $\tau_{k}$.

\subsection{Análisis de rendimiento}

STLQ se centra en ampliar los intervalos de muestreo, acepta cierta degradación en los costos y enfatiza la utilización de recursos. Además, OSIST se enfoca en producir una señal de control a trozos para imitar la entrada de control óptima continua y da importancia al rendimiento de control.

En la Tabla 1 se agrupan las ecuaciones (14) y (15) tomadas desde (Gommans, 2014), y las ecuaciones (16) y (17), desde (Velasco, 2015). Se usan para resumir las directrices de diseño, tanto para las reglas de muestreo $\tau_{k}$, como para la entrada de control a trozos $u_{k}$. A través de estas se pueden identificar los parámetros que afectan el funcionamiento en términos de utilización de recursos y rendimiento de control.

Tabla 1. Reglas de muestreo y entradas de control por trozos

\begin{tabular}{|c|c|}
\hline STLQ & OSIST \\
\hline$\tau_{k}=\mathcal{M}\left(x_{k}, \tau_{\min }, \beta\right) \quad(14)$ & $\tau_{k}=\mathcal{M}\left(x_{k}, \tau_{\max }, \eta, \alpha\right) \quad(16)$ \\
\hline$u_{k}=Z\left(\tau_{k}, \tau_{\min }\right) x_{k} \quad(15)$ & $u_{k}=Z\left(\tau_{k}\right) x_{k} \quad(17)$ \\
\hline
\end{tabular}




\subsubsection{Condición inicial e intervalo de muestreo promedio}

En STLQ se señala que en ausencia de ruido (caso bajo análisis), cualquier condición inicial a lo largo de un segmento $\{\gamma x \mid \gamma>0\}$, conducirá a una misma secuencia de intervalos de muestreo. Dado que la ganancia del controlador es óptima, la dinámica dominante oscilará, lo que significa que los estados saltarán a través de diferentes rayos. Esto producirá un patrón de muestreo oscilatorio que para una ejecución suficientemente larga tendrá el mismo período promedio, independientemente de la condición inicial. Para una caracterización extendida de las secuencias de muestreo véase (Velasco, 2009).

En la regla de muestreo de OSIST en (12), en estado de equilibrio $\left(x_{k}=0\right)$, los intervalos de muestreo son lo más largos posible $\left(\tau_{k}=\tau_{\max }\right)$. La tendencia consiste en obtener intervalos largos a medida que la dinámica de lazo cerrado se aproxima al equilibrio, así, si el estado inicial ya proporciona un intervalo largo, existe una alta probabilidad que la secuencia trabaje en el tope superior de valores. Por lo tanto, pueden alcanzarse intervalos de muestreo promedio más grandes.

El estado inicial más adecuado será el que para OSIST proporcione el período promedio más corto; el tiempo de ejecución deberá ser lo suficientemente largo como para permitir que ambos enfoques pongan al sistema de lazo cerrado en equilibrio.

\subsubsection{Parámetros de muestreo}

Con el fin de asegurar una comparación equitativa, es necesario que STLQ y OSIST generen intervalos de muestreo con límites inferior y superior. En la regla de muestreo (14), STLQ tiene un límite inferior $\tau_{\text {min }}$, mientras que en (16), OSIST un límite superior $\tau_{\text {max }}$. Por lo tanto, los intervalos de muestreo generados por STLQ deberían ser limitados en valor máximo, y los generados por OSIST, en valor mínimo.

En (14), $\tau_{\min }$ también especifica la granularidad temporal del espacio de búsqueda para $\tau_{c}$ (véase (9)), limitando el conjunto de valores posibles que $\tau_{k}$ puede tomar. Sin embargo, esta limitación no se cumple para (16) donde $\tau_{k}$ puede tomar cualquier valor menor que $\tau_{\max }$. Así, se debe ajustar un valor pequeño para $\tau_{\min }$ en la configuración del experimento.

Es necesario considerar que un intervalo de muestreo corto proporciona mejor rendimiento de control (menor costo), mientras que un intervalo de muestreo largo permite menor demanda de recursos (menor utilización). Alternativamente se puede usar el intervalo de muestreo promedio $\bar{h}$ como una métrica de utilización de recursos, considerando

$$
\bar{h}=\frac{1}{n} \sum_{k=0}^{n-1} \tau_{k}
$$

donde $n$ es el número de intervalos de muestreo en un período de tiempo determinado. Mediante 
la aplicación de restricciones en este valor, se evita distorsionar la operación original de cada regla de muestreo, con el fin de obtener resultados aptos para la comparación.

En (14), considerando que cuando se tiene un muestreo periódico cada $\tau_{\min }$ existe un costo óptimo discreto, el factor de escalamiento $\beta$ especifica una degradación permitida con respecto a este costo. Se ajusta así un grado de suboptimalidad en términos de rendimiento de control, pero intrínsecamente, también se configura la demanda de recursos del controlador auto-disparado. Por lo tanto, al permitir una mayor degradación ( $\beta$ más grande) se obtiene un espacio de búsqueda más amplio para $\tau_{c}$, el cual termina con intervalos de muestreo más largos.

El factor de densidad $\eta$ en (16), regula la frecuencia de muestreo y consecuentemente afecta la utilización de recursos. Considerando que un muestreo más denso ( $\eta$ más pequeño) implica la producción de una señal de control a trozos más cercana a la óptima continua, $\eta$ especifica un grado de suboptimalidad en términos de rendimiento de control.

El parámetro de diseño $\alpha$ en (16), permite realizar un ajuste extra en el rendimiento de control. Esta variable ajusta el muestreo de tal manera que se pueden producir más muestras ante una mayor variación de la derivada de la entrada de control óptimo continuo (Bini, 2014); para $\alpha=0$ se obtiene un muestreo periódico.

\subsubsection{Señales de Control}

Una vez calculado el intervalo de muestreo actual $\tau_{k}$, la entrada de control a trozos $u_{k}$ generada por (15) o (17), difiere solamente en la ganancia $Z(\cdot)$ que se aplica. En la expresión de control para STLQ (11), la ganancia se calcula suponiendo que el resto de muestras después de la muestra actual, será igual a $\tau_{\min }$. En el control de OSIST (13), se supone que el resto de muestras será igual a la muestra actual $\tau_{k}$.

\section{Resultados y Discusión}

Esta sección ilustra numéricamente varias cuestiones discutidas en la Sección 2. Sobre una planta conocida, se aplican los criterios enunciados para evaluar el rendimiento de los dos enfoques de control auto-disparado. Además se presentan los resultados de este análisis comparativo.

\subsection{Planta}

La planta considerada es un sistema LTI que tiene la forma de (1) y consiste en un circuito electrónico doble integrador, tal como se describe en Astrom (1997). Su dinámica se representa a través de

$$
G=\left[\begin{array}{ll}
0 & 1 \\
0 & 0
\end{array}\right], H=\left[\begin{array}{l}
0 \\
1
\end{array}\right]
$$


El costo (4) se caracteriza por las matrices de ponderación

$$
Q=\left[\begin{array}{cc}
20 & 0 \\
0 & 0.01
\end{array}\right], R=[0.05]
$$

cuyos valores han sido elegidos para obtener gráficas lisas. En una función de costo LQR, la matriz $Q$ define los pesos en los estados mientras que la matriz $R$ especifica los pesos en la entrada de control. Se puede consultar Murray (2006) para mayor información sobre el ajuste de estas matrices.

A continuación, en las Figuras 1 y 2 se observa el rendimiento de STLQ y OSIST respectivamente. Las gráficas de tallo en azul (Stems) representan las secuencias de muestreo. En el eje-x se encuentran representados los instantes en que cada muestreo se realiza durante el experimento, mientras que en el eje-y, la longitud de los tallos ilustra la magnitud de cada instante de muestreo $\tau_{k}$. Además, las gráficas en rojo exhiben la evolución de los estados $x$ [1] y $x$ [2] del sistema en (19). Estas representaciones en particular, son curvas paramétricas (con $t_{k}$ como parámetro) que trazan la trayectoria de cada particular solución ( $x[1], x[2])$, cuando el tiempo del experimento t se encuentra dentro del rango $0<\mathrm{t}<\infty$.

\subsection{Evaluación de rendimiento}

En la Figura 1 se ilustra la secuencia oscilante de muestreo de la respuesta natural obtenida con STLQ dada una condición inicial arbitraria $\left(x_{0}[1], x_{0}[2]\right)=\left(10 \cos \left(\frac{\pi}{4}\right), 10 \operatorname{sen}\left(\frac{\pi}{4}\right)\right)$, un intervalo de muestreo mínimo $\tau_{\min }$, y un conjunto de parámetros de diseño $\beta$. Se observa el alcance que tiene $\beta$ sobre la utilización de recursos: valores más pequeños de $\beta$ producen patrones de muestreo más densos (mayor utilización), y viceversa, como se enunció en la Sección 2.2.2. Este mismo efecto de utilización se puede advertir a través de la métrica de intervalo de muestreo promedio $\bar{h}$ en (18). Además, los retratos de fase ilustran la convergencia de los estados de la planta hacia el punto de equilibrio $(x[1], x[2])=(0,0)$, indicando estabilidad del sistema a pesar de las diferentes densidades del muestreo.

La Figura 2 muestra el patrón de muestreo (que primero oscila y luego se estabiliza) de la respuesta natural obtenida a través de la estrategia OSIST, considerando la misma condición inicial que la usada para evaluar STLQ (Figura 1), un intervalo de muestreo máximo $\tau_{\max }$, un parámetro de distribución de muestras $\alpha$, y un conjunto de valores de densidad de la secuencia de muestreo $\eta$. Es evidente que el valor del parámetro $\eta$ influye en la utilización de recursos, produciendo muestreos más densos cuando su valor es menor, como se mencionó en la Sección 2.2.2. Además, el muestreo promedio $\bar{h}$ es más pequeño para muestreos más densos, corroborando un mayor uso de recursos. En cuanto a los retratos de fase, se puede observar que los estados convergen al punto de equilibrio indistintamente de las secuencias de muestreo, 
confirmando la estabilidad del sistema. Es importante distinguir que conforme los estados tienden al equilibrio, el muestreo tiende a $\tau_{\max }$.
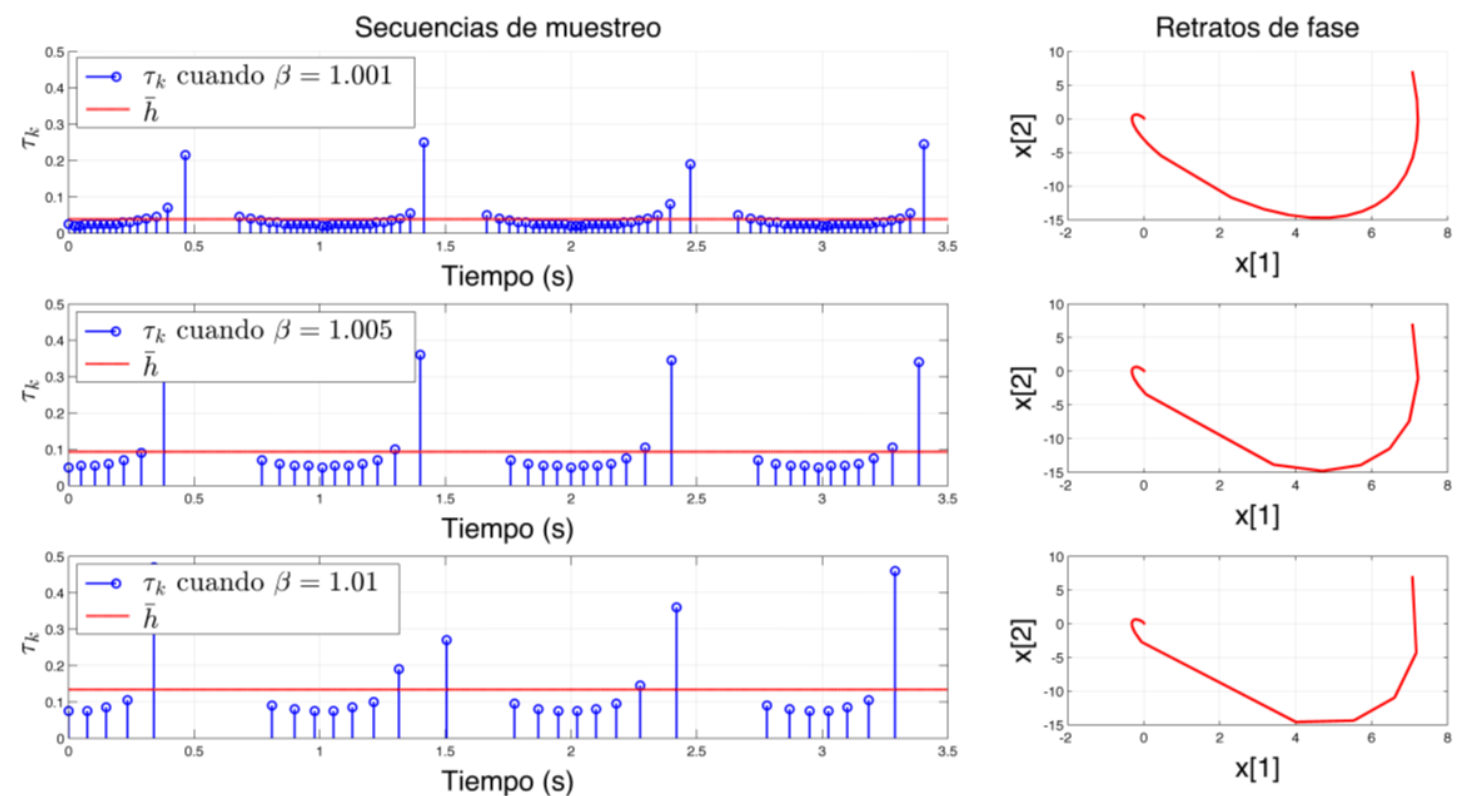

Figura 1. Intervalos de muestreo en STLQ para $\tau_{\min }=0.005 \mathrm{~s}$ y $\beta \in\{1.001,1.005,1.01\}$
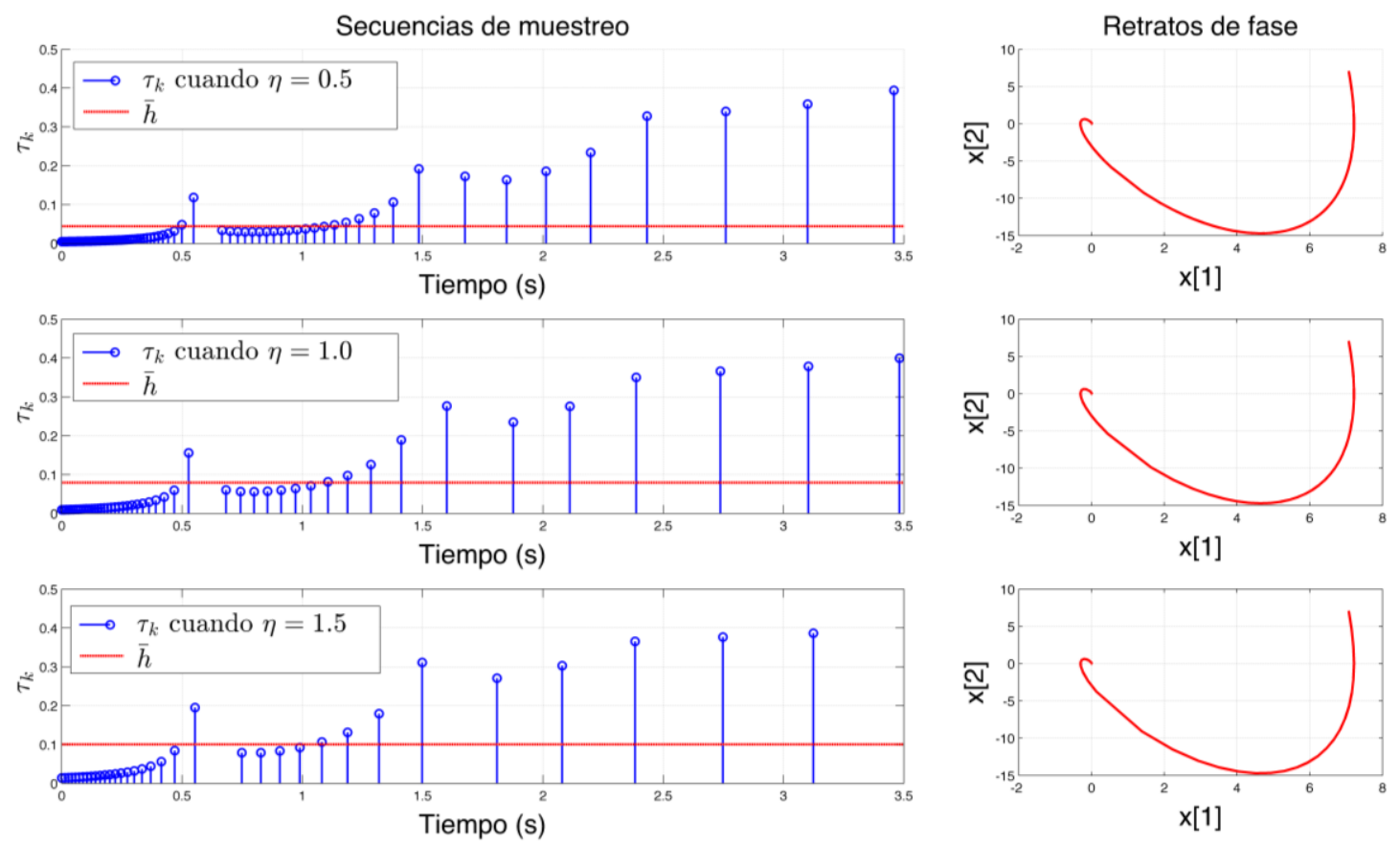

Figura 2. Intervalos de muestreo en OSIST para $\tau_{\max }=0.4 \mathrm{~s}, \alpha=0.67$ y $\eta \in\{0.5,1.0,1.5\}$

En la Figura 3 se analiza el impacto del estado inicial sobre el desempeño de cada enfoque, considerando al intervalo de muestreo promedio como función de la orientación inicial de los estados (véase la Sección 2.2.1). Cada aproximación estabiliza la planta ante diferentes condiciones iniciales expresadas en términos de magnitud constante y orientación variable (se muestran solo ángulos dentro del intervalo $[0, \pi]$ ya que la segunda mitad del círculo es simétrica con respecto a la primera mitad). Si se observa la Figura 3, se obtiene menor utilización (mayor muestreo promedio) para el intervalo de orientaciones $[1.5,2]$, en las dos reglas de muestreo. 
Finalmente, tanto en STLQ como en OSIST el intervalo de muestreo promedio depende de la condición inicial.

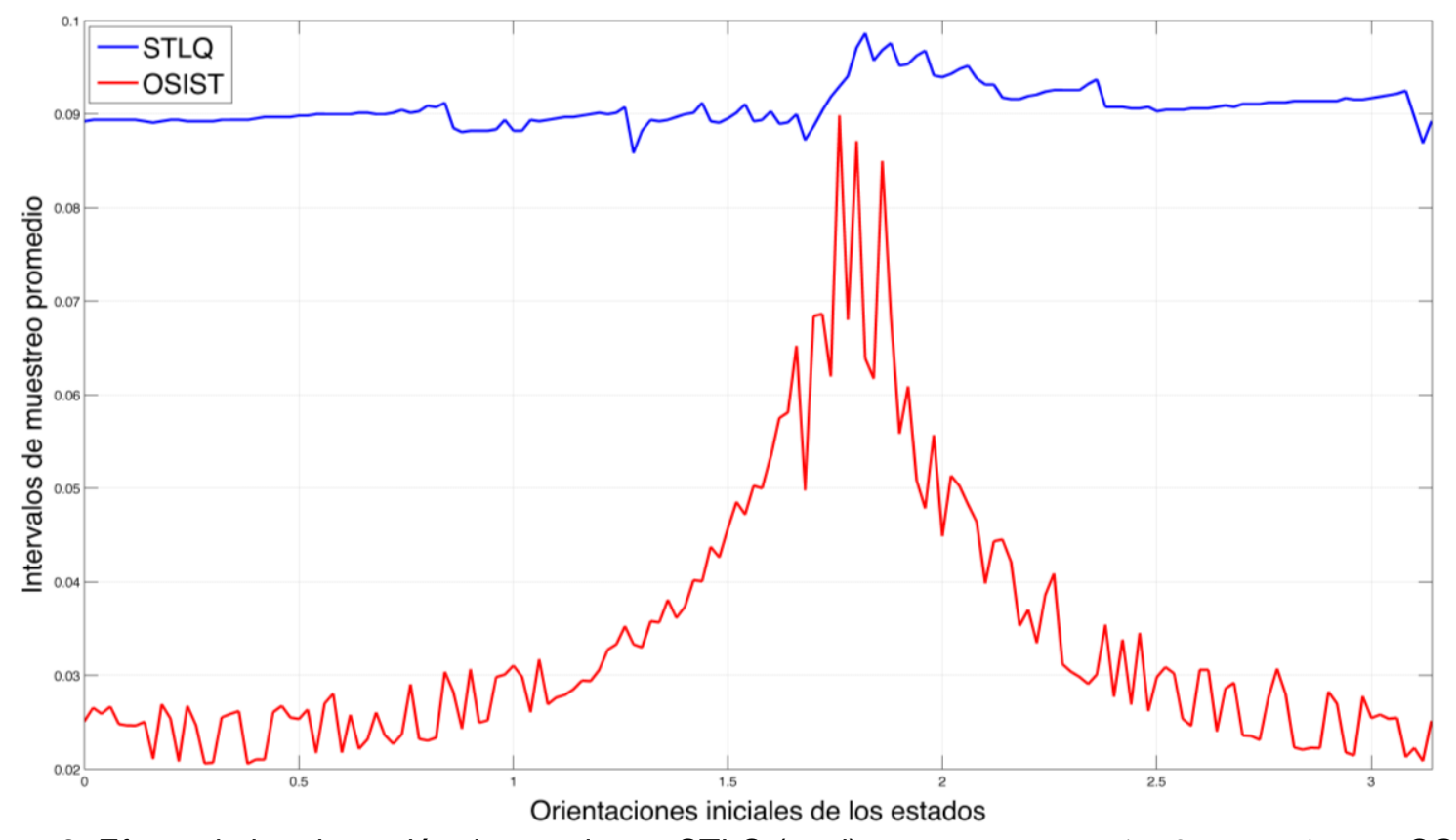

Figura 3. Efecto de la orientación de estado, en STLQ (azul), con $\tau_{\min }=0.005 \mathrm{~s}, \beta=1.005$, y en OSIST (rojo), con $\tau_{\text {max }}=0.4, \eta=1.5$ y $\alpha=0.67$ para OSIST

La Figura 4 muestra el impacto que tienen $\beta$ y $\eta$ en el rendimiento de control. Para ello se utiliza como métrica el costo normalizado, definido como

$$
J_{N}=\frac{J_{\tau}-J_{\infty}}{J_{\infty}}
$$

donde $J_{\infty}$ es el costo LQR mínimo del sistema en tiempo continuo (4), y $J_{\tau}$ es el costo discreto en (8) pero con intervalos de muestreo variables. En la misma Figura 4, la línea negra corresponde al costo obtenido a través del control LQR periódico en tiempo discreto, para diferentes períodos de muestreo. Como puede observarse, el desempeño del controlador periódico disminuye a medida que el período de muestreo aumenta. La curva en azul corresponde a STLQ y la curva en rojo a OSIST, para diferentes parámetros $\beta$ y $\eta$ respectivamente, con configuraciones y estados iniciales arbitrarios. Si se tiene en cuenta que $\beta$ y $\eta$ indican la densidad de la discretización, se evidencia que para valores pequeños, ambos enfoques proporcionan tanto intervalos de muestreo promedio cortos (mayor utilización), como menores costos. Las dos aproximaciones superan al caso periódico.

Un posible espacio para investigar dentro del enfoque STLQ (Gommans, 2014) consistiría en, cumpliendo la misma restricción en el costo, no solo considerar el intervalo de muestreo actual, sino más de un intervalo de muestreo. Además, una nueva alternativa podría explorar si la ocurrencia de intervalos cortos de muestreo adicionales, mejoraría el costo sin poner en riesgo la utilización. Lo anterior se enuncia considerando que ampliar los intervalos de muestreo tanto como sea posible, no es una garantía para lograr un costo mínimo. 


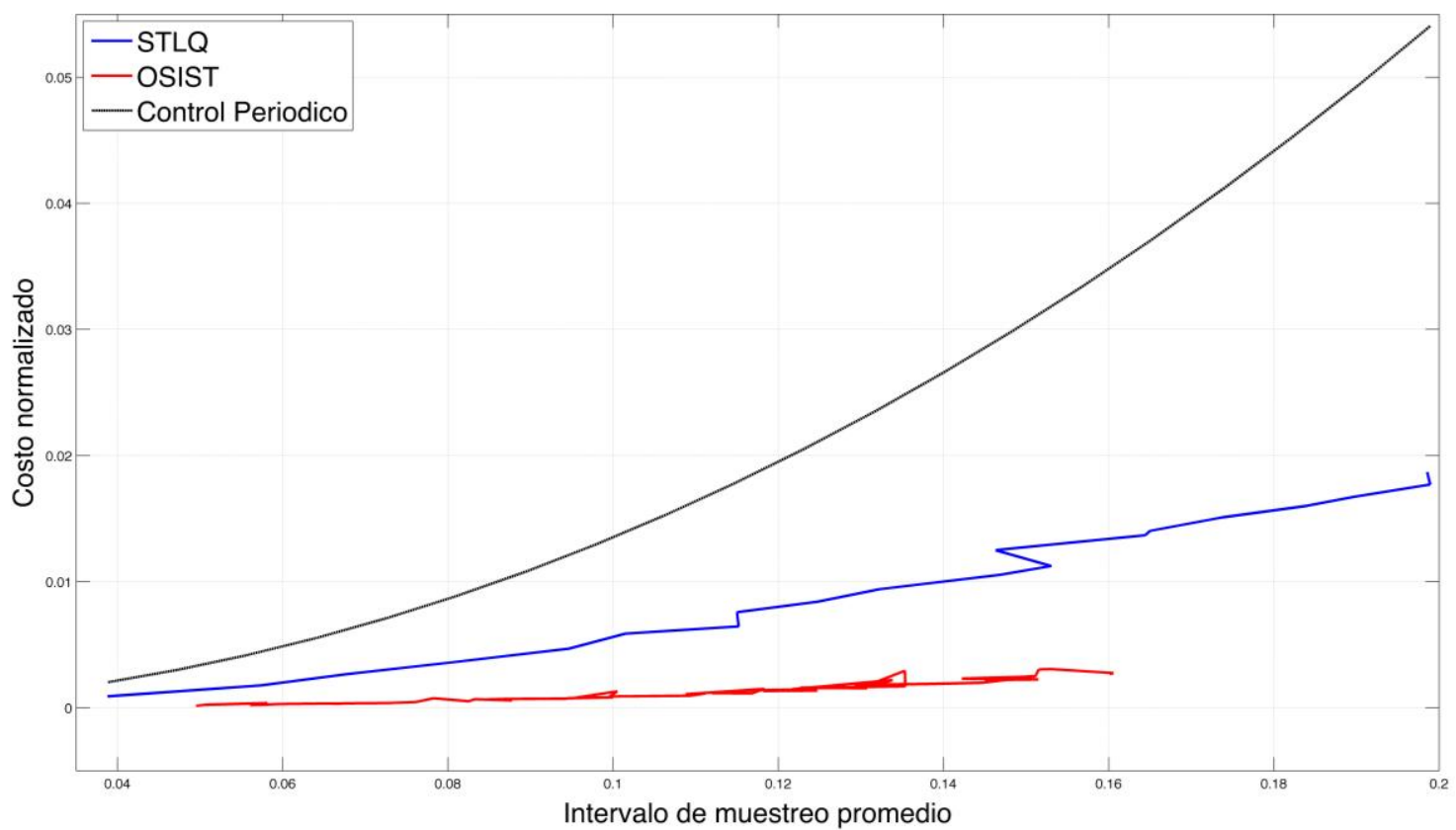

Figura 4. Efecto del factor de escala $\beta$ en STLQ (azul) para $\tau_{\min }=0.005 \mathrm{~s}$ y $\beta \in\{1.001,1.002, \ldots, 1.020\}$, y efecto del factor de densidad $\eta$ en OSIST (rojo) para $\tau_{\max }=0.4 \mathrm{~s}, \alpha=0.67 \mathrm{y} \eta \in\{0.75,0.80, \ldots, 1.7\}$

En OSIST (Velasco, 2015), dado que el costo normalizado bordea cero (Figura 4), y que el algoritmo para ajustar los parámetros de diseño todavía está incompleto, investigaciones futuras podrían explorar el efecto que estos parámetros ejercen sobre el costo. La sencillez de la regla de muestreo (12) facilita su implementación, pero introduce un grado de suboptimalidad cuando se utiliza la aproximación de primer orden de la dinámica de lazo cerrado. Por lo tanto, en estudios posteriores, podría considerarse el uso de aproximaciones de orden superior.

Como trabajo futuro sería factible la formulación de una búsqueda de los ajustes de control que minimicen el costo sujeto a una restricción en demanda de recursos (común para los dos enfoques). Se puede considerar al intervalo promedio de muestreo como una restricción adecuada, utilizando condiciones iniciales que proporcionen al menos un intervalo promedio similar entre las dos aproximaciones.

\section{Conclusiones y Recomendaciones}

Se ha presentado un análisis comparativo de dos enfoques de control auto-disparado que siguen principios de diseño distintos. Este estudio ha demostrado que: ambos tienen un desempeño que supera al del muestreo periódico y queda campo por explorar en la formulación de métricas y condiciones de trabajo aplicables en su evaluación conjunta.

\section{Bibliografía}

Arzén, K. (1999). A Simple Event-Based PID Controller. IFAC World Conference, 18, pp. 423-428.

Recuperado de http://portal.research.lu.se/portal/files/6083100/8521976.pdf 
Astrom, K., \& Bernhardsson, B. (1999). Comparison of periodic and event based sampling for firstorder stochastic systems. 14th IFAC World Congress. Recuperado de https://lup.lub.lu.se/search/ws/files/6208782/8520116.pdf

Astrom, K., \& Wittenmark, B. (1997). Computer-Controlled System. Theory and Design. Third edition. Prentice Hall.

Bini, E., and Buttazzo, G. (2014). The optimal sampling pattern for linear control systems. IEEE Transactions on Automatic Control, 59(1), pp. 78-90, doi:

https://doi.org/10.1109/TAC.2013.2279913

Gommans, T. (2015). Resource-aware control and estimation: an optimization-based approach. Eindhoven University of Technology Library. Recuperado de https://pure.tue.nl/ws/files/12652231/20160118_Gommans.pdf

Gommans, T., Antunes, D., Donkers, T., Tabuada, P., and Heemels, M. (2014). Self-triggered linear quadratic control. Automatica, 50(4), pp. 1279-1287, http://dx.doi.org/10.1016/j.automatica.2014.02.030

Heemels, W., Johansson, K., \& Tabuada, P. (2012). An introduction to event-triggered and selftriggered control. IEEE 51st Annual Conference on Decision and Control (CDC), pp. 3270-3285, doi: https://doi.org/10.1109/CDC.2012.6425820

Meng, X., and Chen, T. (2012). Optimal sampling and performance comparison of periodic and event based impulse control. IEEE Transactions on Automatic Control, 57(12), pp. 3252-3259. doi: https://doi.org/10.1109/TAC.2012.2200381

Molin, A., and Hirche, S. (2013). On the optimality of certainty equivalence for event-triggered control systems. IEEE Transactions on Automatic Control, 58(2), pp. 470-474. doi: https://doi.org/10.1109/TAC.2012.2206719

Murray, R.M. (2006). Control and Dynamical Systems: LQR Control. California Institute of Technology. Recuperado de http://www.cds.caltech.edu/ murray/courses/cds110/wi06/lqr.pdf

Rabi, M., Johansson, K., \& Johansson, M. (2008). Optimal stopping for event-triggered sensing and actuation. 47th IEEE Conference on Decision and Control, pp. 3607-3612. doi: https://doi.org/10.1109/CDC.2008.4739489

Velasco, M., Fuertes, J., \& Martí, P. (2003). The self triggered task model for real-time control systems. IEEE 24th Real-Time Systems Symposium, pp. 67-70. Recuperado de http://citeseerx.ist.psu.edu/viewdoc/download?doi=10.1.1.164.3868\&rep=rep1\&type=pdf 
Velasco, M., Martí, P., and Bini, E. (2009). Equilibrium sampling interval sequences for eventdriven controllers. 2009 European Control Conference, pp. 3773-3778. Recuperado de http://ieeexplore.ieee.org/document/7074987/

Velasco, M., Martí, P., and Bini, E. (2015). Optimal-sampling-inspired self triggered control. 1st IEEE International Conference on Event-based Control, Communication, and Signal Processing, pp. 1-8, doi: https://doi.org/10.1109/EBCCSP.2015.7300648

Velasco, M., Martí, P., Yépez, J., Ruiz, F., Fuertes, J., and Bini, E. (2011). Qualitative analysis of a one-step finite-horizon boundary for event-driven controllers. 50th IEEE Conference on Decision and Control and European Control Conference, pp. 1662-1667. doi:

https://doi.org/10.1109/CDC.2011.6161106 